\title{
Topology and the Dirac Operator Spectrum in Finite-Volume Gauge Theories
}

\author{
P.H. DAMGAARD \\ The Niels Bohr Institute \\ Blegdamsvej 17 \\ DK-2100 Copenhagen \\ Denmark
}

August 17, 2018

\begin{abstract}
The interplay between between gauge-field winding numbers, $\theta$-vacua, and the Dirac operator spectrum in finite-volume gauge theories is reconsidered. To assess the weight of each topological sector, we compare the mass-dependent chiral condensate in gauge field sectors of fixed topological index with the answer obtained by summing over the topological charge. Also the microscopic Dirac operator spectrum in the full finite-volume Yang-Mills theory is obtained in this way, by summing over all topological sectors with the appropriate weight.
\end{abstract}

NBI-HE-99-07

hep-th/9903096 


\section{Introduction}

Although the chiral $\mathrm{SU}_{L}\left(N_{f}\right) \times \mathrm{SU}_{R}\left(N_{f}\right)$ symmetry of QCD is not spontaneously broken in a finite volume, it has long been known that many low-energy aspects of finite-volume QCD nevertheless can be derived from an effective chiral Lagrangian [1]. This is because the dominating degrees of freedom can still be the "would-be" $N_{f}^{2}-1$ pseudo-Goldstone bosons (which are simply called pions in what follows) of the presumed chiral symmetry breaking pattern of $\mathrm{SU}_{L}\left(N_{f}\right) \times \mathrm{SU}_{R}\left(N_{f}\right) \rightarrow \mathrm{SU}\left(N_{f}\right)$ in the infinite volume limit. Clearly at least two scales are of relevance here: if the linear extent $L$ of the four-volume $V$ is much larger than the Compton wavelength $1 / m_{\pi}$ of the pions, the effect of the finite volume is mild, and can be treated as a small correction to the low-energy results of the theory in infinite volume. What is at first sight much more surprising is the fact that even in the opposite limit, when the pion Compton wavelenght much exceeds the size of the box, the effective partition function still becomes of a very simple chiral Lagrangian type. The procedure to derive this effective theory is to treat the zero modes of the pion field as new collective coordinates in the low-energy expansion. When all the dust has settled, the resulting leading-order Lagrangian coincides with what one would have obtained by the naive procedure of simply discarding all derivative terms in the infinite-volume chiral Lagrangian [1]. Independently of whether chiral symmetry is spontanenously broken or not, the pseudoscalar states we here generically denote by pions are guaranteed, by exact mass inequalities, to be the lightest flavor non-singlet mesons in QCD [2].

Naively one might think that an effective Lagrangian technique as described above can provide information only about the chiral order parameter $\Sigma \equiv\langle\bar{\psi} \psi\rangle$ itself, or just soft pion dynamics. However, as shown by Leutwyler and Smilga [3], low-energy constraints on finite-volume gauge theories can, remarkably, be used to provide very non-trivial information about quark and gluon degrees of freedom as well. Specifically, the large-volume identification of the original QCD partition function and the effective Lagrangian restricted to the region

$$
\frac{1}{\Lambda_{Q C D}} \ll L \ll \frac{1}{m_{\pi}},
$$

carries a lot of exact information about the spectral correlators of the smallest eigenvalues of the Dirac operator. While this holds for the full partition functions involved, the scoop of ref. [3] was to note that the analysis simplifies considerably if one focuses on gauge field sectors of fixed topological charge $\nu$. The effective partition function then becomes analytically solvable. It is thus possible to do a direct analytical comparison between, on one side, the full QCD partition function, and, on the other side, the effective partition function. One of the most striking outcomes of this analysis was the derivation of exact spectral sum rules for the Dirac operator in QCD and QCD-like gauge theories [3, 4 .

Surprisingly, the analysis of the spectral properties of the Dirac operator in finite-volume gauge field theories at fixed topological charge [3] has turned out to have an alternative description in terms of Random Matrix Theory [5, 6]. The root of this equivalence lies in the universality of the Random Matrix Theory predictions: there is a huge class of Random Matrix Theories that, in the appropriate scaling limit, yield exactly the same spectral correlation functions [7, 8, 10, 11]. Remarkably, so-called chiral versions of the three classical Random Matrix Theory ensembles have been shown by Verbaarschot to provide precisely the needed three universality classes of chiral symmetry breaking in gauge theories with different gauge groups and different color representations of the fermions 12]. While there are obvious resemblances here to the classification of critical behavior in terms of universality classes in statistical mechanics, the analogy should not be pushed too far. There is no renormalization group at work here, and the way QCD fits into one of these three universality classes 
is by means of algebra (or, at a deeper level, symmetry) rather than dynamics. Indeed, within the last year it has become well understood how all the universal predictions derived from Random Matrix Theory can be rephrased in terms of the effective QCD partition function, suitably extended with additional quark species [13]. Even universality of the results can easily be understood directly from the universality of the Random Matrix Theory partition functions [14] and the equivalence between the effective chiral Lagrangian and the specific Random Matrix Theory with gaussian distribution, which was established in [5]. By means of partially quenched chiral Lagrangians, it is now also possible to derive directly from the effective QCD partition function the microscopic spectral density of the Dirac operator, without any bypass through Random Matrix Theory at all [15]. All these more recent results permit us to say much more about the microscopic Dirac operator spectrum in finite-volume gauge theories. It is therefore appropriate to reconsider the analysis of ref. [3] in this new light.

It is thus partly by historical coincidence that almost all analytical predictions about the microscopic Dirac operator spectrum and the mass-dependent chiral condensate have been derived in gauge sectors of fixed winding number $\nu$. From the point of view of the "real" theory, where one sums over all topological sectors, this is analogous to knowing only the Fourier modes of a periodic function. While it may be interesting to study just these Fourier modes (and in the case of QCD much can indeed be learned from them), we would still rather like to know the full function. For QCD this means that we should know the predictions for the full theory at any given value of the vacuum angle $\theta$, or, at least, since there are very stringent experimental bounds on the magnitude of this CP-violating $\theta$-angle, for the specific value of $\theta=0$.

The problem of summing over all winding numbers becomes particularly important when we consider tests of the analytical predictions for QCD sectors of fixed topological charge $\nu$ by means of lattice gauge theory. Because lattice gauge configurations are not smooth on the scale of the lattice spacing, the very definition of gauge field topology is from the beginning an ambiguous concept. With so-called staggered fermions there is no help from index theorems (which otherwise can serve as an alternative definition of topological sectors) because there are no guaranteed exact zero modes away from the continuum limit [16]. In fact, lattice gauge theory data for QCD with staggered fermions and no restrictions on lattice field topology show remarkably good agreement with the analytical predictions for just the sector of zero winding number, $\nu=0$. This phenomenon was first observed in comparisons of lattice data with analytical formulas for the mass-dependent chiral condensate [17], and later precisely the same effect was seen in lattice simulations of the microscopic Dirac operator spectrum with staggered fermions [18, 19. One can argue that just because staggered fermions have no exact zero modes, this explains the puzzle. Are present-day lattice gauge theory simulations with staggered fermions thus only probing the sector of zero topological charge? Despite the problem mentioned above, lattice gauge field configurations generated by conventional Monte Carlo algorithms cannot all be classified as topologically trivial. They contain a distribution of configurations that smoothly fall into the precise topological sectors of the continuum theory as the lattice cut-off is decreased. Of course, in a given Monte Carlo sampling the sector corresponding to $\nu=0$ will be dominant. To understand the significance of just these configurations we need to know not only the analytical predictions for given sectors of fixed topological index, but also the weight with which these different topological sectors are contributing to full QCD. Very recently it has become feasible to address this in lattice gauge theory [21, 22]. But in the finite-volume region (1), these questions can also be answered analytically. For QCD-like theories the relevant partition functions at fixed topological

\footnotetext{
${ }^{1}$ Based on lattice data with dynamical quark masses so large on the relevant scale that the simulations effectively were quenched.

${ }^{2}$ In this connection, see the recent careful studies by the SCRI and Columbia groups [20].
} 
charge $\nu$ are known in closed analytical form [24]. It is simple to show that these fixed- $\nu$ partition functions precisely give the non-trivial weights with which each fixed- $\nu$ observable contributes to the full answer. Using this fact, we assess in section 2 the importance of the $\nu=0$ sector in bulding up the mass-dependent chiral condensate of the full theory. Section 3 is devoted to the microscopic spectral density of the Dirac operator, and we carry out the analogous procedure of summing over topological charges. Interestingly, one of the simple conclusions is that in the massless case the microscopic spectral density of the Dirac operator in the full theory simply equals that of the $\nu=0$ sector. This holds for all $N_{f} \geq 2$. The case $N_{f}=1$ is - as expected - special, and there are then additional zero-mode contributions to the full microscopic spectral density from just the two sectors of $\nu=+1$ and $\nu=-1$. In the massless limit just these two zero-mode contributions to the spectral density provide the required non-vanishing condensate for $N_{f}=1$, even in this finite-volume situation. By numerically performing the sum over topological charges, we show how the full microscopic spectral density changes away from the $\nu=0$ prediction as the mass is increased for different values of $N_{f}$. We point out a difficulty with taking the quenched limit, and briefly discuss the more exotic chiral symmetry breaking cases, which correspond to different symmetries of the Dirac operator. All of the predictions described here can be tested in lattice gauge theory simulations. In fact, one of our main points is precisely that one can simultaneously test both the detailed analytical predictions in fixed- $\nu$ sectors, and the predicted weight with which these sectors build up the full answer (which, in principle, should be much easier to measure). Section 5 contains our conclusions.

\section{Summing over Winding Numbers}

We begin with a few basic definitions. Consider QCD in Euclidean space on a finite 4-dimensional torus. Its four-volume can be taken to be just $L^{4}$, or $L^{3} / T$. The former case leads in the $L \rightarrow \infty$ limit to ordinary zero-temperature QCD, while in the latter case, if we take $T$ fixed, the $L \rightarrow \infty$ limit gives Euclidean QCD at (small[1) temperature T. For our purposes there is no need to distinguish between the two cases, as the infinite (three-)volume limit of the chiral condensate just must be chosen appropriately. The four-volume is in both cases denoted by $V$. As discussed at length in e.g. refs. [3, 25], gauge fields on such a four-torus can be described by a gauge potential $A_{\mu}(x)$ on the full Euclidean space, plus a transition function $\Omega(x)$, which describes the transition from one periodic cell to another (possibly non-trivial because $A_{\mu}(x)$ need only be periodic modulo gauge transformations). On the lattice one often prefers to work with strictly periodic gauge link variables (and hence trivial transition function), but by a gauge transformation these links can be made equivalent to a set of link variables with a non-trivial transition function. In the continuum such a gauge transformation will, however, necessarily be singular. Non-trivial winding numbers arise precisely from non-trivial transition functions [25].

For an $\mathrm{SU}\left(N_{c}\right)$ gauge theory with $N_{f}$ fermions in the fundamental representation, antiperiodic boundary conditions on the four-torus for these fermions the topological charge

$$
\nu=\frac{1}{32 \pi^{2}} \int d^{4} x \operatorname{Tr}\left[F_{\mu \nu} F_{\rho \sigma}\right] \epsilon_{\mu \nu \rho \sigma}
$$

runs over integer values. With a $\theta$-term in the action, the partition function thus reads

$$
\mathcal{Z}(\theta)=\sum_{\nu=-\infty}^{\infty} e^{i \nu \theta} \int[d A]_{\nu} \prod_{f} \operatorname{det}\left[i \not D-m_{f}\right] e^{S_{Y M}[A]},
$$

\footnotetext{
${ }^{3}$ The temperature must be bounded by $m_{\pi} \ll T \ll \Lambda_{Q C D}$, in complete analogy with the condition (1).
} 
after integrating out the fermions. Alternatively,

$$
\mathcal{Z}(\theta)=\sum_{\nu=-\infty}^{\infty} e^{i \nu \theta} \mathcal{Z}_{\nu},
$$

where $\mathcal{Z}_{\nu}$ is like a Fourier transform of the full partition function:

$$
\mathcal{Z}_{\nu}=\frac{1}{2 \pi} \int_{0}^{2 \pi} d \theta e^{-i \nu \theta} \mathcal{Z}(\theta)
$$

Consider first the case $N_{f} \geq 2$, and assume that chiral symmetry in the infinite-volume limit breaks spontaneously according to $\mathrm{SU}_{L}\left(N_{f}\right) \times \mathrm{SU}_{R}\left(N_{f}\right) \rightarrow \mathrm{SU}\left(N_{f}\right)$. As shown in ref [1], the effective partition function then takes on a very simple form in the finite-volume region (11):

$$
\mathcal{Z}(\theta)=\int_{S U\left(N_{f}\right)} d U \exp \left[V \Sigma \operatorname{Re}\left[e^{i \theta / N_{f}} \operatorname{Tr} \mathcal{M} U^{\dagger}\right]\right]
$$

where $\mathcal{M}$ is the quark mass matrix 7 , and $\Sigma$ is the infinite-volume chiral condensate (for the massless theory with $\theta=0$ ). One of the main lessons to learn from the analysis of ref. [3] is that these finite-volume effective partition functions for $N_{f} \geq 2$ in surprisingly many ways resemble the finitevolume partition function with $N_{f}=1$. In fact, although the case $N_{f}=1$ is radically different in that the axial U(1) symmetry is broken by the anomaly rather than spontaneously, the effective partition function turns out be given by what one naively could have extrapolated from (6), namely the simple exponential [3]

$$
\mathcal{Z}(\theta)=\exp \left[V \Sigma \operatorname{Re}\left[e^{i \theta} m\right]\right] .
$$

In all cases the effective partition functions depend only on the combination $\mu_{i} \equiv \Sigma V m_{i}$. Keeping $\mu_{i}$ fixed as the four-volume $V$ is taken to infinity thus entails taking the chiral limit in a correlated manner. Moreover, the bound (1) must be satisfied throughout. In statistical mechanics such a procedure is known as finite-size scaling, and the $\mu_{i}$ are finite-size scaling variables.

The Fourier coefficients $\mathcal{Z}_{\nu}$ have been computed analytically for all $N_{f}$ [3, 24]. For $N_{f} \geq 2$ they can conveniently be written

$$
\mathcal{Z}_{\nu}\left(\left\{\mu_{i}\right\}\right)=\frac{\operatorname{det} \mathcal{A}\left(\left\{\mu_{i}\right\}\right)}{\Delta\left(\mu^{2}\right)}
$$

where the $N_{f} \times N_{f}$ matrix $\mathcal{A}\left(\left\{\mu_{i}\right\}\right)$ is $\left(I_{n}(x)\right.$ is the modified Bessel function of order $\left.n\right)$

$$
\mathcal{A}\left(\left\{\mu_{i}\right\}\right)=\mu_{i}^{j-1} I_{\nu+j-1}\left(\mu_{i}\right),
$$

and

$$
\Delta\left(\mu^{2}\right)=\prod_{i<j}\left(\mu_{i}^{2}-\mu_{j}^{2}\right)
$$

is the Vandermonde determinant. With an obvious interpretation of the formula (8), it even includes the case $N_{f}=1$, for which [3]

$$
\mathcal{Z}_{\nu}(\mu)=I_{\nu}(\mu) .
$$

One notices that the general expression (8) is symmetric in $\nu$,

$$
\mathcal{Z}_{\nu}\left(\left\{\mu_{i}\right\}\right)=\mathcal{Z}_{-\nu}\left(\left\{\mu_{i}\right\}\right),
$$

as follows also directly from the original definition, eqs. (5) and (6).

\footnotetext{
${ }^{4}$ From now on we always take this mass matrix to be real and diagonal: $\mathcal{M}=\operatorname{diag}\left(m_{1}, \ldots, m_{N_{f}}\right)$.
} 


\subsection{The Chiral COndensate For $N_{f}=1$}

Having already observed that in the limit (1) the relevant scaling variables are $\mu_{i}=\Sigma V m_{i}$, we from now on try to eliminate as many factors of $V$ and $\Sigma$ as possible. We hence measure all mass-dependent chiral condensates $\Sigma\left(\theta ;\left\{\mu_{i}\right\}\right)$ in units of $\Sigma$, and effectively work with a unit four-volume. The massdependent chiral condensate for $N_{f}=1$ is then simply defined by

$$
\Sigma(\theta ; \mu)=\frac{\partial}{\partial \mu} \ln \mathcal{Z}(\theta ; \mu)
$$

In this $N_{f}=1$ case the result is trivial and in fact $\mu$-independent: $\Sigma(\theta ; \mu)=\cos (\theta)$, as follows from

eq. (11)..$^{5}$ In any case, we shall mainly be concerned with the case $\theta=0$, for which, in our units, $\Sigma(0 ; \mu)=1$.

Let us now compare with what we would find if we were to restrict ourselves to gauge field averages over fixed topological charge only. As already observed by Leutwyler and Smilga [3], this restriction is quite drastic. By definition,

$$
\Sigma_{\nu}(\mu)=\frac{\partial}{\partial \mu} \ln \mathcal{Z}_{\nu}(\mu)
$$

which gives

$$
\Sigma_{\nu}(\mu)=\frac{1}{I_{\nu}(\mu)}\left[I_{\nu+1}(\mu)+\frac{\nu}{\mu} I_{\nu}(\mu)\right] .
$$

For $\nu$ positive, the last term can easily be traced to the $|\nu|$ zero modes of the Dirac operator $\not D$ : In terms of the non-zero Dirac eigenvalues $\lambda_{n}$ the original partition function (3) reads

$$
\mathcal{Z}(\theta)=\sum_{\nu=-\infty}^{\infty} \prod_{f}\left(m_{f}\right)^{|\nu|} e^{i \nu \theta} \int[d A]_{\nu} \prod_{f} \prod_{n}\left[\lambda_{n}^{2}+m_{f}^{2}\right] e^{S_{Y M}[A]}
$$

and the last term in (15) precisely matches the term due to the prefactor $m^{|\nu|}$. However, when $\nu$ is negative this interpretation is not correct: in that case the sign of the second term in (15) is just the opposite of what is needed. There is no mystery here, but only a slight complication which we shall return to in section 3.1. Indeed, from the very definition (14) and the property (12), the mass-dependent chiral condensate (15) is seen to be symmetric in $\nu: \Sigma_{\nu}(\mu)=\Sigma_{-\nu}(\mu)$. Therefore the obvious $\sim 1 / \mu$ singularity in eq. (15) for $\nu>0$ is actually matched by a singularity of exactly the same strength for $\nu<0$.

We start by considering the case $\theta=0$. In fig. 1 we show $\Sigma_{\nu}(\mu)$ for a few small values of $\nu$, and compare each of them with the full answer $\Sigma(\mu)$. Contrary to the full answer which in this finitevolume limit is just the trivial constant $\Sigma=1$, the chiral condensates in individual fixed- $\nu$ sectors have non-trivial mass dependences. In this case we certainly cannot conclude that the $\nu=0$ sector adequately reproduces the full answer. But fixed $-\nu$ chiral condensates also show another disturbing feature, which we already mentioned above: for $\nu \neq 0$ they diverge in the limit $\mu \rightarrow 0$. Actually, the second term in eq. (15) by itself does not contribute to the full sum over topological sectors. To see this, we first derive a simple formula that tells us how to relate the full chiral condensate $\Sigma(\theta ; \mu)$ to the chiral condensates $\Sigma_{\nu}(\mu)$ in sectors of fixed topological index:

$$
\Sigma(\theta ; \mu)=\frac{\partial}{\partial \mu} \ln \mathcal{Z}(\theta ; \mu)
$$

\footnotetext{
${ }^{5}$ The $\theta$-dependence of the condensate is not interesting; for $\theta \neq 0$ it simply shifts from $\langle\bar{\psi} \psi\rangle$ to $\left\langle\bar{\psi} \gamma^{5} \psi\right\rangle[3]$.
} 


$$
\begin{aligned}
& =\frac{\partial}{\partial \mu} \ln \left(\sum_{\nu=-\infty}^{\infty} \mathcal{Z}_{\nu}(\mu) e^{i \nu \theta}\right) \\
& =\frac{1}{\mathcal{Z}(\theta ; \mu)} \sum_{\nu=-\infty}^{\infty} e^{i \nu \theta} \frac{\partial}{\partial \mu} \mathcal{Z}_{\nu}(\mu) \\
& =\frac{1}{\mathcal{Z}(\theta ; \mu)} \sum_{\nu=-\infty}^{\infty} e^{i \nu \theta} \mathcal{Z}_{\nu}(\mu) \Sigma_{\nu}(\mu) \\
& =\left\langle\left\langle\Sigma_{\nu}(\mu)\right\rangle\right\rangle .
\end{aligned}
$$

We have here defined an "average over topology" by

$$
\langle\langle F(\nu)\rangle\rangle \equiv \frac{1}{\mathcal{Z}(\theta ; \mu)} \sum_{\nu=-\infty}^{\infty} e^{i \nu \theta} \mathcal{Z}_{\nu}(\mu) F(\nu) .
$$

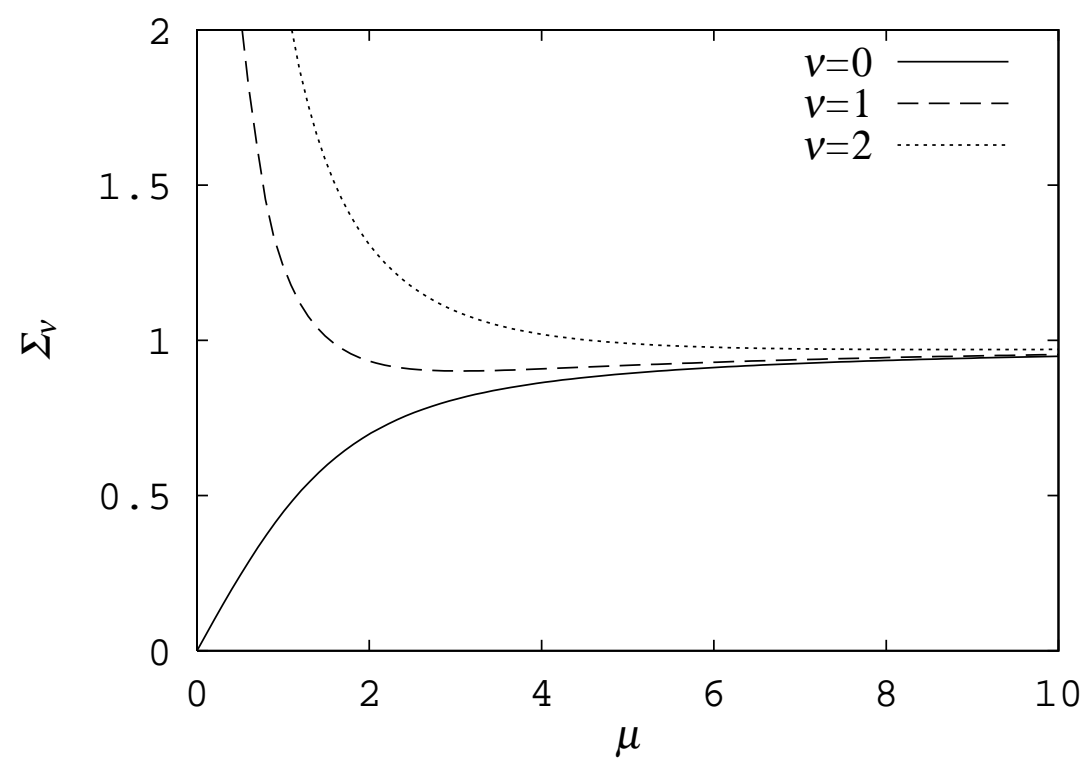

Figure 1: The mass-dependent chiral condensate $\Sigma_{\nu}(\mu)$ for $N_{f}=1$. Shown are the results for a few fixed values of $\nu$. Even for $\theta=0$ they are quite different from the full answer (the constant 1 in that case).

The relation (17) tells us with which weight the chiral condensates in fixed topological sectors contribute to the full answer: Each $\Sigma_{\nu}(\mu)$ comes with weight factor $e^{i \nu \theta} Z_{\nu}(\mu)$. In fig. 2 we show how the full answer, the constant $\Sigma=1$ for $\theta=0$, is built up as we sum over topological charge $\nu$ from a given $-|\nu|_{\max }$ to $+|\nu|_{\max }$ in eq. (17). Perhaps surprisingly, we find that the $\nu=0$ contribution alone is not very significant, never exceeding more than around $20 \%$ of the full answer. In the shown range of $\mu$ we also see that by summing from, say, $\nu=-8$ to $\nu=+8$ we essentially obtain the complete $\Sigma$. The divergences that appear in $\Sigma_{\nu}(\mu)$ for $\nu \neq 0$ are obviously completely removed when one performs the required average over topology. In the present case of $\theta=0$ this can easily be seen analytically. According to eqs. (15) and (17), and using $\mathcal{Z}(\theta=0 ; \mu)=\exp [\mu]$, we here have

$$
\begin{aligned}
\Sigma(\theta=0 ; \mu) & =e^{-\mu} \sum_{\nu=-\infty}^{\infty}\left[I_{\nu+1}(\mu)+\frac{\nu}{\mu} I_{\nu}(\mu)\right] \\
& =e^{-\mu} \sum_{\nu=-\infty}^{\infty} I_{\nu+1}(\mu) .
\end{aligned}
$$






Figure 2: The full mass-dependent chiral condensate $\Sigma(\theta=0, \mu)$ for $N_{f}=1$. The exact answer equals 1 . Shown are the results of summing over the first few values of $\nu$. The $\nu=0$ sector is not particularly significant.

The second term in the first line actually does not contribute at all: This is obvious for $\nu=0$, and for $\nu \neq 0$ the contribution from $+\nu$ precisely cancels the contribution from $-\nu$. Next, substituting the Bessel function identity

$$
\sum_{\nu=-\infty}^{\infty} e^{i \nu \theta} I_{\nu}(\mu)=e^{\mu \cos (\theta)},
$$

we of course recover the correct result $\Sigma(\theta=0 ; \mu)=1$ from eq. (19).

For a non-zero vacuum angle $\theta$ the $\nu / \mu$-term in $\Sigma_{\nu}(\mu)$ does not disappear as simply when we sum over different topological sectors. Again this $N_{f}=1$ case is very illustrative. Now, using some simple Bessel function identities, we have

$$
\begin{aligned}
\Sigma(\theta ; \mu) & =e^{-\mu \cos (\theta)} \sum_{\nu=-\infty}^{\infty} e^{i \nu \theta}\left[I_{\nu+1}(\mu)+\frac{\nu}{\mu} I_{\nu}(\mu)\right] \\
& =\frac{1}{2} e^{-\mu \cos (\theta)} \sum_{\nu=-\infty}^{\infty} e^{i \nu \theta}\left[I_{\nu+1}(\mu)+I_{\nu-1}(\mu)\right] \\
& =\cos (\theta) .
\end{aligned}
$$

where in the last line we have agin used eq. (20). In this case the second term in (15) is certainly required to obtain the right answer from the sum (17). More generally, we also learn from eq. (17) that the $\mu \rightarrow 0$ singularities in $\Sigma_{\nu}(\mu)$ for $\nu \neq 0$ are killed in the sum over topological sectors because these divergences are weighted by $e^{i \nu \theta} Z_{\nu}(\mu)$. In general, $Z_{\nu}(\mu) \sim \mu^{N_{f}|\nu|}$ for small $\mu$. This follows both explicitly from the representation (6), or, in the original QCD language, from the Dirac determinant and the index theorem. Thus only for $N_{f}=1$, and in that case only for the sectors with $\nu= \pm 1$, can these $1 / \mu$ singularities in $\Sigma_{\nu}(\mu)$ give any non-zero contribution to the massless chiral condensate. In fact, one easily checks that in this case just these two contributions precisely yield the finite value $\Sigma(\theta ; 0)=\cos (\theta)$, as required [3]. 


\subsection{MORE FlaVors: $N_{f} \geq 2$.}

It is convenient to first restrict ourselves to the case of equal masses, $\mu_{i}=\mu, \forall i$, so that the chiral condensates of all $N_{f}$ species become equal. Then

$$
\Sigma(\theta ; \mu) \equiv \frac{1}{N_{f}} \frac{\partial}{\partial \mu} \ln \mathcal{Z}(\theta ; \mu)=\left\langle\left\langle\Sigma_{\nu}(\theta ; \mu)\right\rangle\right\rangle
$$

with $\Sigma_{\nu}(\theta ; \mu)$ defined analogously. Consider first the case $N_{f}=2$. The full partition function is known analytically [3]:

$$
\mathcal{Z}(\theta ; \mu)=\frac{\sqrt{2}}{\mu \sqrt{(1+\cos (\theta))}} I_{1}\left(\mu\left(2(1+\cos (\theta))^{1 / 2}\right)\right. \text {. }
$$

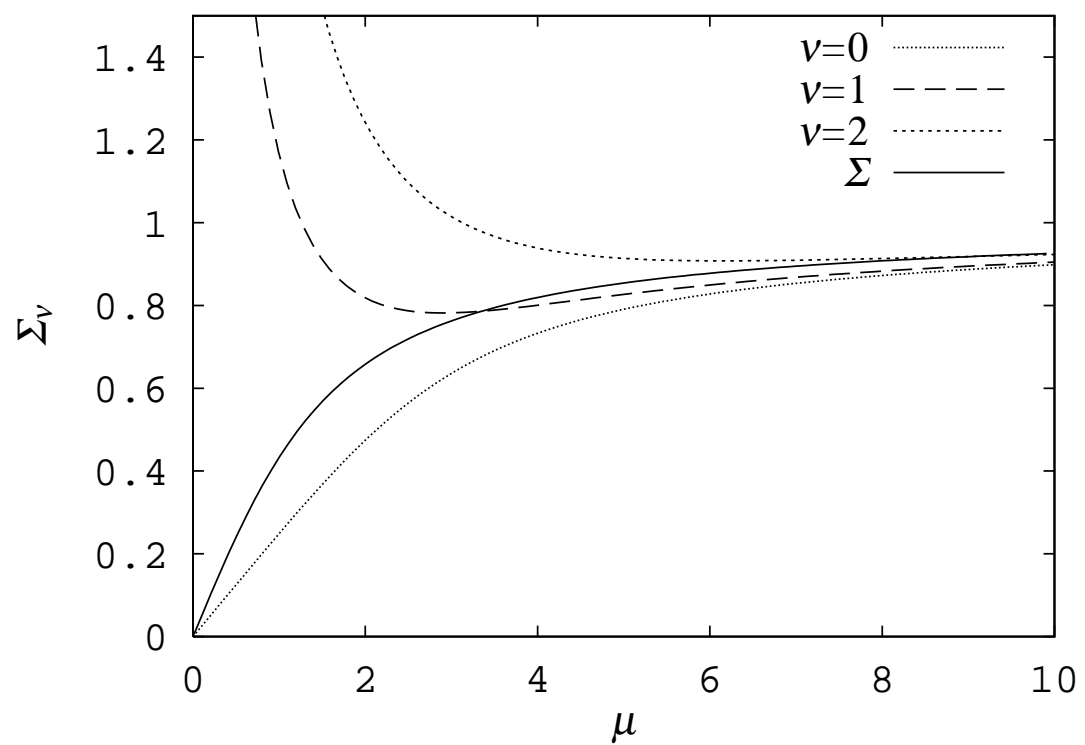

Figure 3: The chiral condensate $\Sigma_{\nu}(\mu)$ for $N_{f}=2$ and equal masses $\mu$. There is no qualtitative difference with the picture from the $N_{f}=1$ theory. For comparison we have also displayed the full chiral condensate $\Sigma(\mu)$ for $\theta=0$.

Let us again first restrict ourselves to the case $\theta=0$. The full mass-dependent chiral condensate then becomes

$$
\Sigma(\theta=0 ; \mu)=\frac{I_{0}(2 \mu)+I_{2}(2 \mu)}{2 I_{1}(2 \mu)}-\frac{1}{2 \mu},
$$

which, despite appearances, is not singular at $\mu=0$. The partition function (8) reads

$$
Z_{\nu}(\mu)=I_{\nu}(\mu)^{2}-I_{\nu+1}(\mu) I_{\nu-1}(\mu),
$$

which gives

$$
\Sigma_{\nu}(\mu)=\frac{1}{2}\left\{\frac{I_{\nu}(\mu) I_{\nu+1}(\mu)-I_{\nu-1}(\mu) I_{\nu+2}(\mu)}{I_{\nu}(\mu)^{2}-I_{\nu+1}(\mu) I_{\nu-1}(\mu)}+\frac{\nu}{\mu}\right\} .
$$

We note again the presence of an explicit $\nu / \mu$-term from the zero modes for $\nu \geq 0$ (in which case the first term in (26) indeed remains finite in the limit $\mu \rightarrow 0$ ). This term will always be present in $\Sigma_{\nu}(\mu)$, 
for any $N_{f}$. It reads in general $\nu /\left(N_{f} \mu\right)$, and thus contributes

$$
\frac{1}{N_{f}} \frac{1}{\mu} \sum_{\nu=-\infty}^{\infty} \nu Z_{\nu}(\theta=0 ; \mu)
$$

to $\Sigma(\theta=0 ; \mu)$. Using the symmetry (12), this is seen to vanish: At $\theta=0$ this contribution to $\Sigma(\theta ; \mu)$ always sums up to zero, for any $N_{f}$. When $\theta \neq 0$ this is no longer true:

$$
\begin{aligned}
\frac{1}{N_{f}} \frac{1}{\mu} \sum_{\nu=-\infty}^{\infty} \nu e^{i \nu \theta} Z_{\nu}(\theta ; \mu) & =\frac{-i}{N_{f}} \frac{1}{\mu} \frac{\partial}{\partial \theta} \sum_{\nu=-\infty}^{\infty} e^{i \nu \theta} Z_{\nu}(\mu) \\
& =\frac{-i}{N_{f}} \frac{1}{\mu} \frac{\partial}{\partial \theta} Z(\theta ; \mu) .
\end{aligned}
$$

In particular, for the case at hand, $N_{f}=2$, we find that this equals

$$
\frac{i \sin (\theta)}{2 \mu(1+\cos (\theta))} I_{2}\left(\mu\left(2(1+\cos (\theta))^{1 / 2}\right)\right.
$$

(which indeed vanishes at $\theta=0$ ). Moreover, we notice that this term (27) is always strictly imaginary. Because the full sum is guaranteed to be real, this means that in some sense even for $\theta \neq 0$ the contribution of this term to $\Sigma_{\nu}(\theta ; \mu)$ is only apparent; it only serves as to remove a corresponding imaginary part from the rest of the sum, leaving a remaining real answer.

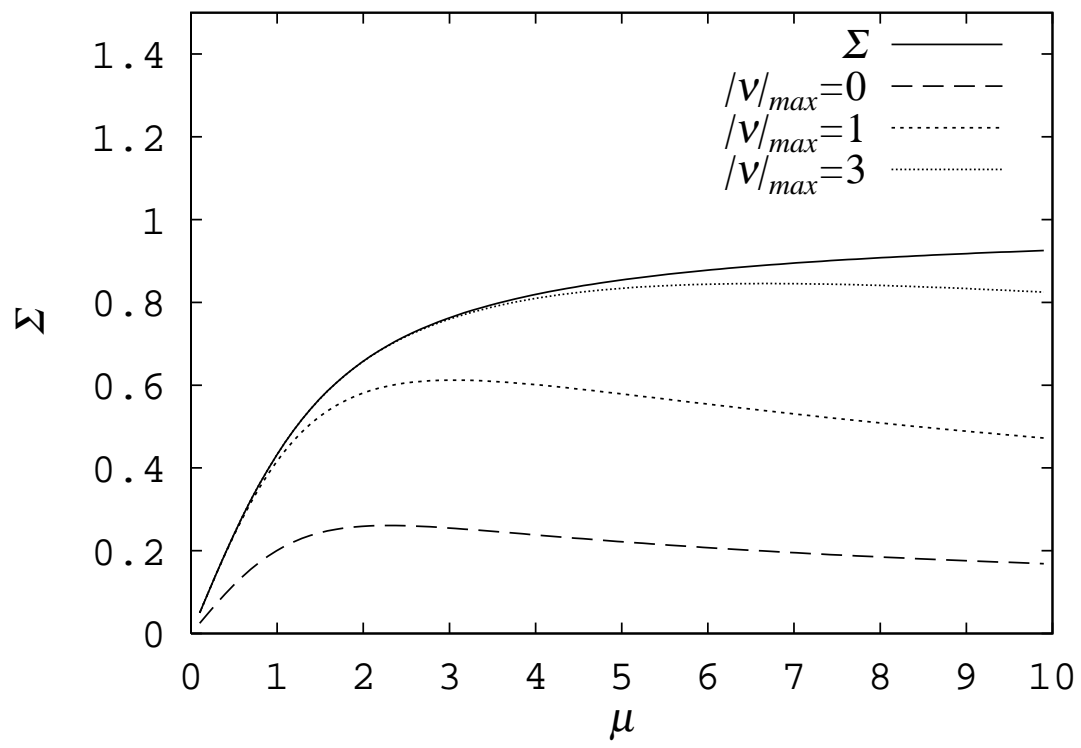

Figure 4: The full chiral condensate $\Sigma(\mu)$ for $N_{f}=2, \theta=0$, and equal masses $\mu$. We again compare different approximations, by summing only over the first few topoloigcal sectors. The $\nu=0$ sector is again not particularly significant.

We now check to which degree of accuracy the chiral condensate $\Sigma(\theta ; \mu)$ for $N_{f}=2$ and $\theta=0$ (i.e. eq. (24)) coincides with just the chiral condensate in fixed- $\nu$ sectors (26). This is shown in fig. 3 for a few small values of $\nu$. The divergent zero-mode contributions of course make the condensates for $\nu \neq 0$ very different from the complete answer. And actually in this $N_{f}=2$ case the chiral condensate in just the $\nu=0$ sector at least has the qualitatively right behavior. But again, to see to what extent 
the different topological sectors contribute to the full answer, we must add up the different sectors with the right weight. In fig. 4 we show how the full chiral condensate (24) is built up as we sum over topological charge $\nu$ from given $-|\nu|_{\max }$ to $+|\nu|_{\max }$ in eq. (22). As in the $N_{f}=1$ case, we find that the $\nu=0$ contribution alone is not particularly significant at all. It is obviously a generic feature, which is not specific to the very special circumstances of $N_{f}=1$. The cases with $N_{f} \geq 3$ are indeed not qualitatively different [26], and we do not display any of them here.

\section{The Microscopic Spectral Density}

Eigenvalues $\lambda_{n}$ of the Dirac operator are defined by $i \not D \phi_{n}=\lambda_{n} \phi_{n}$, and the spectral density is then

$$
\bar{\rho}\left(\lambda ;\left\{\mu_{i}\right\}\right) \equiv\left\langle\sum_{n} \delta\left(\lambda-\lambda_{n}\right)\right\rangle
$$

where the average includes the sum over topological sectors. Because the weighting is done with the fermion determinant, the density depends on the masses $\mu_{i}$ too. The double-microscopic limit is taken by keeping all $\mu_{i}=V \Sigma m_{i}$ and $\zeta=V \Sigma \lambda$ fixed as $V \rightarrow \infty$. The definition (28) of course extends to averages in fixed- $\nu$ sectors as well:

$$
\bar{\rho}^{(\nu)}\left(\lambda ;\left\{\mu_{i}\right\}\right) \equiv\left\langle\sum_{n} \delta\left(\lambda-\lambda_{n}\right)\right\rangle_{\nu}
$$

We now go to the double-microscopic limit. As for any other gauge-field expectation value in fixed- $\nu$ sectors, also the microscopic spectral density $\bar{\rho}_{S}^{(\nu)}\left(\zeta ;\left\{\mu_{i}\right\}\right)$ can be summed to give the microscopic spectral density of the full theory:

$$
\bar{\rho}_{S}\left(\zeta ; \theta,\left\{\mu_{i}\right\}\right)=\mathcal{Z}\left(\theta ;\left\{\mu_{i}\right\}\right)^{-1} \sum_{\nu=-\infty}^{\infty} e^{i \nu \theta} \mathcal{Z}_{\nu}\left(\left\{\mu_{i}\right\}\right) \bar{\rho}_{S}^{(\nu)}\left(\zeta ;\left\{\mu_{i}\right\}\right)
$$

It is useful to separate out the zero-modes explicitly:

$$
\begin{aligned}
\bar{\rho}_{S}\left(\zeta ; \theta,\left\{\mu_{i}\right\}\right) & =\mathcal{Z}\left(\theta ;\left\{\mu_{i}\right\}\right)^{-1} \sum_{\nu=-\infty}^{\infty} e^{i \nu \theta} \mathcal{Z}_{\nu}\left(\left\{\mu_{i}\right\}\right)\left[\rho_{S}^{(\nu)}\left(\zeta ;\left\{\mu_{i}\right\}\right)+|\nu| \delta(\zeta)\right] \\
& \equiv \rho_{S}\left(\zeta ; \theta,\left\{\mu_{i}\right\}\right)+\langle\langle|\nu|\rangle\rangle \delta(\zeta)
\end{aligned}
$$

with an obvious definition of $\rho_{S}\left(\zeta ; \theta,\left\{\mu_{i}\right\}\right)$.

It has been shown 13, 15] that the microscopic spectral density (29) without zero-mode contributions can be expressed directly in terms of the effective partition function,

$$
\rho_{S}^{(\nu)}\left(\zeta ;\left\{\mu_{i}\right\}\right)=\frac{1}{2}(-1)^{\nu}|\zeta| \prod_{f=1}^{N_{f}}\left(\zeta^{2}+\mu_{f}^{2}\right) \frac{\mathcal{Z}_{\nu}^{\left(N_{f}+2\right)}\left(\left\{\mu_{i}\right\}, i \zeta, i \zeta\right)}{\mathcal{Z}_{\nu}^{\left(N_{f}\right)}\left(\left\{\mu_{i}\right\}\right)}
$$

where we for clarity have explicitly indicated the number of fermion species that are involved on the right hand side. Actually, from the connection to Random Matrix Theory it is also known that all microscopic spectral correlators $\rho_{S}^{(\nu)}\left(\zeta_{1}, \ldots, \zeta_{n} ;\left\{\mu_{i}\right\}\right)$ can be derived from just one "master formula" [13],

$$
K_{S}^{(\nu)}\left(\zeta, \zeta^{\prime} ; \mu_{1}, \ldots, \mu_{N_{f}}\right)=\frac{1}{2}(-1)^{\nu} \sqrt{\zeta \zeta^{\prime}} \prod_{f=1}^{N_{f}} \sqrt{\left(\zeta^{2}+\mu_{f}^{2}\right)\left(\zeta^{\prime 2}+\mu_{f}^{2}\right)} \frac{\mathcal{Z}_{\nu}^{\left(N_{f}+2\right)}\left(\left\{\mu_{i}\right\}, i \zeta, i \zeta^{\prime}\right)}{\mathcal{Z}_{\nu}^{\left(N_{f}\right)}\left(\left\{\mu_{i}\right\}\right)} .
$$


All spectral correlation functions follow from this single function:

$$
\rho_{S}^{(\nu)}\left(\zeta_{1}, \ldots, \zeta_{n} ;\left\{\mu_{i}\right\}\right)=\operatorname{det}_{a, b} K_{S}^{(\nu)}\left(\zeta_{a}, \zeta_{b} ;\left\{\mu_{i}\right\}\right)
$$

where the determinant on the right hand side is taken in terms of the $n \times n$ matrix in the arguments $\zeta_{a}, \zeta_{b}$.

The full microscopic spectral density without zero-mode contributions, $\rho_{S}\left(\zeta ; \theta,\left\{\mu_{i}\right\}\right)$, takes a particularly simple form in the massless limit. Using $\mathcal{Z}_{0}(0)=1$ and $\mathcal{Z}_{\nu}(0)=0$ for all $\nu \neq 0$, we immediately get

$$
\rho_{S}\left(\zeta ; \theta,\left\{\mu_{i}=0\right\}\right)=\rho_{S}^{(\nu=0)}\left(\zeta ;\left\{\mu_{i}=0\right\}\right) .
$$

That is, in the massless limit the full microscopic spectral density without zero modes simply coincides with the corresponding density of the $\nu=0$ sector. Note in particular that in this massless limit the full microscopic spectral density is independent of the vacuum angle $\theta$. This is as expected: in QCD with at least one massless flavor any $\theta$-angle can be removed by a chiral rotation.

The result (35) may seem to contradict the fact that in the $N_{f}=1$ theory the chiral condensate is $\Sigma(\theta ; \mu=0)=\cos (\theta)$ even though the massless limit is taken at finite volume. Indeed, even at finite volume there is a kind of Banks-Casher relation for the microscopic spectral density too,

$$
\lim _{\mu \rightarrow 0}(2 \mu) \int_{0}^{\infty} d \zeta \frac{\rho_{S}(\zeta ; \theta, \mu)}{\zeta^{2}+\mu^{2}}=\pi \rho_{S}(0 ; \theta, 0)=\pi \rho_{S}^{(\nu=0)}(0 ; 0)=0 .
$$

This microscopic spectral density alone can therefore never give rise to a condensate in the massless finite-volume theory. But the full condensate potentially also receives contributions from the zero modes, even at zero mass. This part equals (taking $N_{f}$ equal masses for simplicity)

$$
\begin{aligned}
\left(N_{f} \mu\right)^{-1}\langle\langle|\nu|\rangle\rangle & =\left[N_{f} \mu \mathcal{Z}\left(\theta ;\left\{\mu_{i}\right\}\right)\right]^{-1} \sum_{\nu=-\infty}^{\infty} e^{i \nu \theta} \mathcal{Z}_{\nu}\left(\left\{\mu_{i}\right\}\right)|\nu| \\
& =2\left[N_{f} \mu \mathcal{Z}\left(\theta ;\left\{\mu_{i}\right\}\right)\right]^{-1} \sum_{\nu=1}^{\infty} \cos (\nu \theta) \mathcal{Z}_{\nu}\left(\left\{\mu_{i}\right\}\right) \nu
\end{aligned}
$$

In the limit $\mu \rightarrow 0$ this expression vanishes for all $N_{f} \geq 2$. In view of (36) this just means that for $N_{f} \geq 2$ there are no chiral condensates in the massless limit, in agreement with the fact that there cannot be spontaneous symmetry breaking in these finite-volume theories. For $N_{f}=1$ and $\theta=0$ the sum in (37) can be performed analytically, giving [3]

$$
\mu^{-1}\langle\langle|\nu|\rangle\rangle=e^{-\mu}\left[I_{0}(\mu)+I_{1}(\mu)\right],
$$

whose limit at $\mu=0$ indeed equals unity, as required in order to obtain $\Sigma=1$ in that case. In general, for $\theta \neq 0$ we note again that because of the behavior $I_{n}(x) \sim x^{|n|}$ as $x \rightarrow 0$, only the $\nu= \pm 1$ terms contribute to the sum when $\mu \rightarrow 0$, yielding precisely the required $\mu^{-1}\langle\langle|\nu|\rangle\rangle \rightarrow \cos (\theta)$ in the limit $\mu \rightarrow 0$.

Inserting the formula (32) into the definition of $\rho_{S}\left(\zeta ; \theta,\left\{\mu_{i}\right\}\right)$ in eq. (31) gives an interesting compact expression for the full microscopic spectral density:

$$
\rho_{S}\left(\zeta ; \theta,\left\{\mu_{i}\right\}\right)=\mathcal{Z}^{\left(N_{f}\right)}\left(\theta ;\left\{\mu_{i}\right\}\right)^{-1} \sum_{\nu=-\infty}^{\infty} e^{i \nu \theta} \mathcal{Z}_{\nu}\left(\left\{\mu_{i}\right\}\right) \rho_{S}^{(\nu)}\left(\zeta ;\left\{\mu_{i}\right\}\right)
$$




$$
\begin{aligned}
& =\frac{1}{2}|\zeta| \prod_{f=1}^{N_{f}}\left(\zeta^{2}+\mu_{f}^{2}\right) \mathcal{Z}^{\left(N_{f}\right)}\left(\theta ;\left\{\mu_{i}\right\}\right)^{-1} \sum_{\nu=-\infty}^{\infty} e^{i \nu \theta}(-1)^{\nu} \mathcal{Z}_{\nu}^{\left(N_{f}+2\right)}\left(\left\{\mu_{i}\right\}, i \zeta, i \zeta\right) \\
& =\frac{1}{2}|\zeta| \prod_{f=1}^{N_{f}}\left(\zeta^{2}+\mu_{f}^{2}\right) \frac{\mathcal{Z}^{\left(N_{f}+2\right)}\left(\theta+\pi ;\left\{\mu_{i}\right\}, i \zeta, i \zeta\right)}{\mathcal{Z}^{\left(N_{f}\right)}\left(\theta ;\left\{\mu_{i}\right\}\right)} .
\end{aligned}
$$

While this formula strongly resembles the one for fixed $\nu$, we note the effect of the $(-1)^{\nu}$-factor in the individual $\rho_{S}^{(\nu)}\left(\lambda ;\left\{\mu_{i}\right\}\right)$-contributions: The partition function with two additional fermion species (in the numerator) is to be evaluated at a shifted vacuum angle: $\theta+\pi$. Thus even if we insist on getting the physical microscopic spectral density with $\theta=0$ (or at least exceedingly small), we need to know also the effective QCD partition function with two more flavors, and with a non-vanishing vacuum angle.

One can perform a similar summation over topological charge of all microscopic spectral correlators $\rho_{S}^{(\nu)}\left(\zeta_{1}, \ldots, \zeta_{n} ;\left\{\mu_{i}\right\}\right)$. However, the representation (33) does not give a a convenient compact expression, due to the fact that the determinant must be taken inside the summation over $\nu$. Fortunately, there exists a different expression for $\rho_{S}^{(\nu)}\left(\zeta_{1}, \ldots, \zeta_{n} ;\left\{\mu_{i}\right\}\right)$ (see the third paper of ref. [13]), which, although more unwieldy in general when $\nu$ is fixed, is more convenient in this context:

$$
\begin{aligned}
\rho_{S}^{(\nu)}\left(\zeta_{1}, \ldots, \zeta_{k} ;\left\{\mu_{i}\right\}\right)= & C^{(k)} \prod_{i}^{k}\left(\zeta_{i} \prod_{f=1}^{N_{f}}\left(\zeta_{i}^{2}+\mu_{f}^{2}\right)\right) \prod_{j<l}^{k}\left(\zeta_{j}^{2}-\zeta_{l}^{2}\right)^{2} \\
& \times \frac{\mathcal{Z}_{\nu}^{\left(N_{f}+2 k\right)}\left(\left\{\mu_{i}\right\},\left\{i \zeta_{1}\right\}, \ldots,\left\{i \zeta_{k}\right\}\right)}{\mathcal{Z}_{\nu}^{\left(N_{f}\right)}\left(\left\{\mu_{i}\right\}\right)},
\end{aligned}
$$

Summing this expression over $\nu$ will yield the microscopic spectral correlators of the full theory, now expressed in terms of the ratio of a partition function of $N_{f}+2 k$ species and the usual partition function corresponding to the actual number of fermions $N_{f}$.

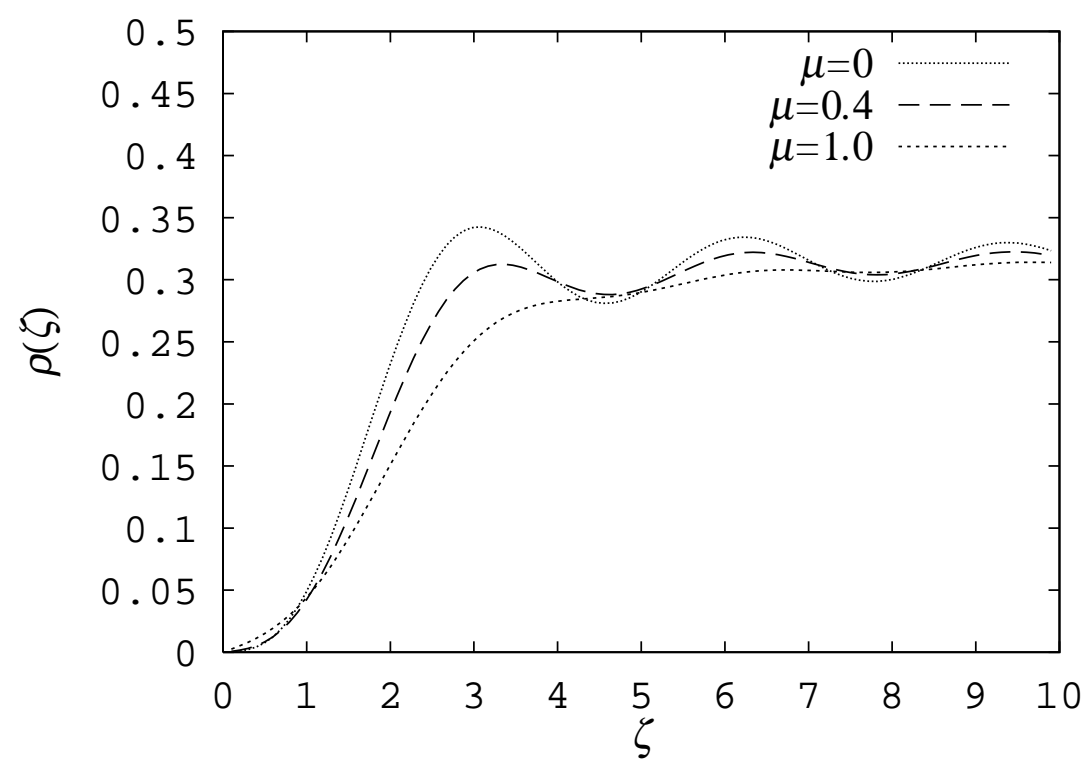

Figure 5: The full microscopic spectral density of the $N_{f}=1$ theory with $\theta=0$. The massless case coincides with the $\nu=0$ result. 
The full effective partition function (6) is unfortunately not known in closed analytical form for $N_{f} \geq 3$. So to evaluate the right hand side of eq. (39) it is most convenient to simply perform the sum over topological charges numerically. We know already the full answer in the massless case (35), and it is thus interesting to trace the change in $\rho_{S}\left(\lambda ; \theta,\left\{\mu_{i}\right\}\right)$ as the mass is increased. In fig. 5 we show the full microscopic spectral density for the $N_{f}=1$ theory with $\theta=0$. The $\mu=0$ curve indeed coincides exactly with the massless prediction of just the $\nu=0$ sector 6

$$
\rho_{S}^{(\nu=0)}(\zeta ; \mu=0)=\frac{1}{2}|\zeta|\left[J_{1}(\zeta)^{2}-J_{2}(\zeta) J_{0}(\zeta)\right]
$$

As the mass increases, sectors with higher values of $|\nu|$ begin to contribute. Because the oscillations in $\rho_{S}^{(\nu)}(\zeta ; \mu)$ are shifted to the right as $|\nu|$ increases, there is a certain amount of destructive interference, and the full microscopic spectral density becomes smoother as $\mu$ is increased. The limit $\mu \rightarrow \infty$, which naively should correspond to the quenched theory, is ill-defined, as will be discussed below.

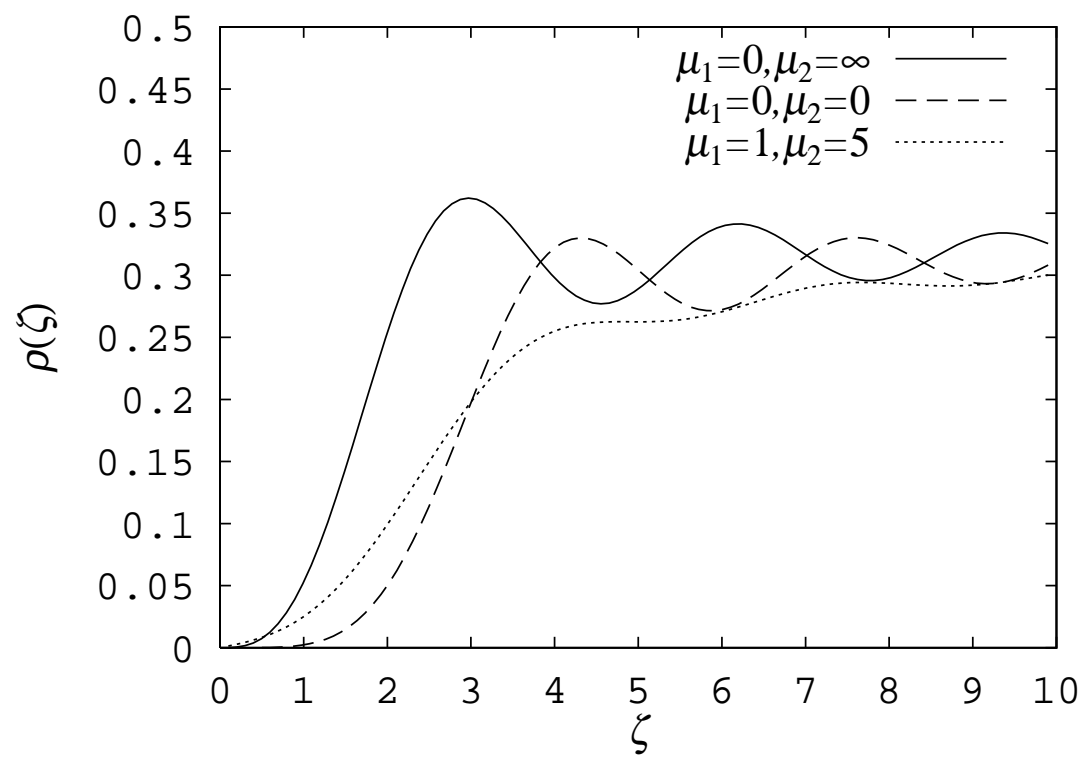

Figure 6: The full microscopic spectral density of the $N_{f}=2$ theory with $\theta=0$. When both fermion masses are set to zero we recover the $\nu=0$ result. When one of the masses is taken to infinity, we recover the full microscopic spectral density of the $N_{f}=1$, by decoupling.

The case $N_{f}=2$ is more interesting. Because of the simple identification (35), the $\mu_{1}=\mu_{2}=0$ limit simply reproduces the massless $N_{f}=2$ density of the just the $\nu=0$ sector,

$$
\rho_{S}^{(\nu=0)}\left(\zeta ; \mu_{1}=\mu_{2}=0\right)=\frac{1}{2}|\zeta|\left[J_{2}(\zeta)^{2}-J_{3}(\zeta) J_{1}(\zeta)\right] .
$$

In another extreme limit, when, say, $\mu_{2} \rightarrow \infty$, this second fermion decouples. If we still take $\mu_{1}=0$, we thus recover the simple massless prediction of just the $\nu=0$ sector of the $N_{f}=1$ theory, eq. (41). For intermediate masses, there is again some amount of destructive interference that smoothes out the microscopic spectral density. Shown in fig. 6 are the two limits mentioned above, and one example of the prediction for intermediate masses. 


\subsection{Careful With the Sign of $\nu$}

We have already noticed that the symmetry (12) under $\nu \rightarrow-\nu$ also implies $\Sigma_{\nu}\left(\left\{\mu_{i}\right\}\right)=\Sigma_{-\nu}\left(\left\{\mu_{i}\right\}\right)$. But this holds only if we consider all contributions, including the $\nu / \mu$-piece. For example, for $N_{f}=1$ (and $\theta=0$ ) the two terms on the right hand side of eq. (15) are not separately invariant under $\nu \rightarrow-\nu$, but the sum is. This has consequences for our definition of the microscopic spectral density $\rho_{S}^{(\nu)}\left(\lambda,\left\{\mu_{i}\right\}\right)$, which does not include the contribution from zero modes.

It follows from eq. (12) and the representation (32) that also

$$
\rho_{S}^{(\nu)}\left(\lambda,\left\{\mu_{i}\right\}\right)=\rho_{s}^{(-\nu)}\left(\lambda,\left\{\mu_{i}\right\}\right)
$$

Clearly also the full microscopic spectral density (29) is thus symmetric under $\nu \rightarrow-\nu$. Let us for illustration consider one of the "massive spectral sum rules" for $N_{f}=1$, which was derived in ref. 266] for the case $\nu \geq 0$. First, by definition,

$$
\begin{aligned}
\Sigma_{\nu}(\mu) & =2 \mu \int_{0}^{\infty} d \lambda \frac{\bar{\rho}_{S}^{(\nu)}(\lambda ; \mu)}{\lambda^{2}+\mu^{2}} \\
& =2 \mu \int_{0}^{\infty} d \lambda \frac{\rho_{S}^{(\nu)}(\lambda ; \mu)}{\lambda^{2}+\mu^{2}}+\frac{|\nu|}{\mu} .
\end{aligned}
$$

Comparing this with eq. (15), we see that there are actually two different massive sum rules, depending on whether $\nu$ is positive or negative:

$$
\begin{array}{ll}
\nu \geq 0: & \int_{0}^{\infty} d \lambda \frac{\rho_{S}^{(\nu)}(\lambda ; \mu)}{\lambda^{2}+\mu^{2}}=\frac{I_{\nu+1}(\mu)}{2 \mu I_{\nu}(\mu)}, \\
\nu \leq 0: & \int_{0}^{\infty} d \lambda \frac{\rho_{S}^{(\nu)}(\lambda ; \mu)}{\lambda^{2}+\mu^{2}}=\frac{I_{\nu+1}(\mu)}{2 \mu I_{\nu}(\mu)}+\frac{\nu}{\mu^{2}} .
\end{array}
$$

It is straightforward to check that in the limit $\mu \rightarrow 0$ these two relations precisely lead to two massless spectral sum rules that can be combined into (the sum running over strictly positive eigenvalues only):

$$
\left\langle\sum_{n}^{\prime} \frac{1}{\lambda_{n}^{2}}\right\rangle_{\nu}=\frac{1}{4(|\nu|+1)},
$$

in complete agreement with ref. [3]. These considerations of course trivially generalize to any $N_{f}$, where only the right hand side of eq. (45) becomes increasingly more complicated [26].

The $N_{f}=1$ case that we have used as an example here is particularly simple also when it comes to an analytical check on our formula for the full microscopic spectral density (39). In that case one can explicitly confirm the relation

$$
\Sigma(\theta ; \mu)=2 \mu \int_{0}^{\infty} d \zeta \frac{\rho_{S}(\theta ; \zeta, \mu)}{\zeta^{2}+\mu^{2}}+\frac{1}{\mu}\langle\langle|\nu|\rangle\rangle
$$

since all the involved integrations can be performed analytically. (The left hand side is of course just the trivial constant $\cos (\theta)$ in this case, and the full spectral density $\rho_{S}(\theta ; \zeta, \mu)$ is indeed just a function that achieves this $\mu$-independence of the right hand side as well). 


\subsection{Trouble With The Quenched Limit}

The quenched limit of $N_{f} \rightarrow 0$ is not easily taken. In gauge-field sectors of fixed topological charge $\nu$, the quenched mass-dependent chiral condensate reads [17, 15]

$$
\Sigma_{\nu}(\mu)=\mu\left(I_{\nu}(\mu) K_{\nu}(\mu)+I_{\nu+1}(\mu) K_{\nu-1}(\mu)\right)+\frac{\nu}{\mu},
$$

where $K_{n}(x)$ is the modified Bessel function. This quenched chiral condensate also satisfies $\Sigma_{\nu}(\mu)=$ $\Sigma_{-\nu}(\mu)$. It shares the $\sim 1 / \mu$ divergence with the unquenched condensates when $\nu \neq 0$. In the latter case we have already seen that this divergence is harmless in the sense that it is killed once we sum over topological charge. Here this is no longer true. If we simply assume that $\mathcal{Z}_{\nu}(\mu)=1$ for all $\nu$ in the quenched limit, it is not even possible to sum over topological charge. There is now no suppression of higher- $\nu$ contributions, and the sum in

$$
\Sigma(\theta ; \mu)=\frac{1}{\mathcal{Z}(\theta ; \mu)} \sum_{\nu=-\infty}^{\infty} e^{i \nu \theta} \Sigma_{\nu}(\mu)
$$

does not converge. If one tries to "regularize" the sum by introducing some maximum topological charge $|\nu|_{\max }$ and defining

$$
\mathcal{Z}(\theta ; \mu)_{\text {reg }} \equiv \sum_{\nu=-|\nu|_{\max }}^{\nu=+|\nu|_{\max }} e^{i \nu \theta} \Sigma_{\nu}(\mu),
$$

eq. (49) still has no well-defined limit as $|\nu|_{\max } \rightarrow \infty$. This failure of convergence is not related to the $\mu \rightarrow 0$ singularities in fixed- $\nu$ sectors; it arises here from the infinite summation over topological charge, if, in the quenched approximation, we assume that each topological sector enters with equal weight $\mathcal{Z}_{\nu}(\mu)=1$. It is clearly invalid to make this assumption. In fact, in the pure gauge theory the topological susceptibility $\chi_{t}=\left\langle\left\langle\nu^{2}\right\rangle\right\rangle$ (in our units) is a well-defined and finite number, and the distribution of topological charge is believed to be Gaussian in the infinite-volume limit [27]:

$$
\frac{\mathcal{Z}_{\nu}}{\mathcal{Z}} \sim \frac{1}{2 \pi \chi_{t}} e^{-\nu^{2} / 2 \chi_{t}}
$$

It is tempting to simply insert this ansatz for $\mathcal{Z}_{\nu}$ into eq. (49) instead of unity, but it is not quite consistent. The whole framework here is based on chiral Ward identities, of which 28]

$$
N_{f}\left\langle\left\langle\nu^{2}\right\rangle\right\rangle=\mu
$$

indeed is a direct consequence of these finite-volume partition functions [3]. Taking the quenched limit detaches us from this tight constraint, and a different analysis is required to establish the behavior of $\mathcal{Z}_{\nu}$ as a function of $\nu$. In any case, the $\mu \rightarrow 0$ singularities are no longer removed by the sum over topological charge, because the distribution of $\mathcal{Z}_{\nu}$ will be $\mu$-independent. This $\sim 1 / \mu$ singularity in the (full) quenched chiral condensate has recently been confirmed by direct lattice measurements using both domain-wall and overlap fermions 20].

With these observations, we should certainly expect difficulties with the quenched microscopic spectral density. The quenched microscopic density in a gauge field sector of fixed $\nu$ reads [6, 17]

$$
\rho_{S}^{(\nu)}(\zeta)=\frac{1}{2}|\zeta|\left[J_{\nu}(\zeta)^{2}-J_{\nu+1}(\zeta) J_{\nu-1}(\zeta)\right]
$$


According to eq. (31), and if we again take $\mathcal{Z}_{\nu}(\mu)$ to be a $\nu$-independent constant (say, unity), the sum in

$$
\rho_{S}\left(\lambda ; \theta,\left\{\mu_{i}\right\}\right)=\mathcal{Z}\left(\theta ;\left\{\mu_{i}\right\}\right)^{-1} \sum_{\nu=-\infty}^{\infty} e^{i \nu \theta} \mathcal{Z}_{\nu}\left(\left\{\mu_{i}\right\}\right) \rho_{S}^{(\nu)}\left(\lambda ;\left\{\mu_{i}\right\}\right)
$$

again requires regularization. Introducing a maximum topological charge $|\nu|_{\max } \rightarrow \infty$ as above, we actually find a remarkable regularity. Let us for convenience restrict ourselves to $\theta=0$. Shown in fig. 7 is a series of successive summations with increasing $|\nu|_{\max }$. The resulting function is approximately linear ๆ, until, at a point that shifts to infinity with increasing $|\nu|_{\text {max }}$, it turns over into the constant value $1 / \pi$. The slope of the linear part thus depends on the choice of $|\nu|_{\max }$, and there is no convergence of the sum. As discussed above, the approximation of taking all $\mathcal{Z}_{\nu}$ to unity is just drastically wrong.



Figure 7: Summing over topological sectors with incorrect weight: if in the quenched theory one simply assumes that $\mathcal{Z}_{\nu}=1$ the resulting microscopic spectral density becomes linear up to the value $1 / \pi$, where it turns flat. This point where the linear part is replaced by a flat part moves to infinity with increasing $|\nu|_{\max }$, and thus has no limit.

Another way of reaching the quenched limit is by decoupling all $N_{f}$ fermions in any given theory by sending all masses to infinity. For simplicity taking $N_{f}=1$, and sending the mass $\mu$ to infinity, we obtain precisely the same kind of curve as obtained above in the naive way of just summing over each fixed- $\nu$ sector with equal weight. The cut-off which controls the point where $\rho_{S}(\zeta)$ turns from linear to flat behavior is now played by this mass $\mu$. This suggests that decoupling all fermions by taking all their rescaled masses $\mu_{i}$ to infinity is not providing a physical limit of the full theory. Indeed, one easily checks that in this extreme limit all partition functions $\mathcal{Z}_{\nu}$ become independent of $\nu$, thus explaining why the two different ways of trying to obtain the quenched microscopic spectral density described here fail in precisely the same manner.

How does this result fit in with the general formula (39)? Taking the $N_{f} \rightarrow 0$ limit in that equation

\footnotetext{
${ }^{6}$ I thank S. Nishigaki for first pointing this out to me.
} 
gives

$$
\rho_{S}(\lambda ; \theta)=\frac{1}{2}|\zeta| \frac{\mathcal{Z}^{(2)}(\theta+\pi ; i \zeta, i \zeta)}{\mathcal{Z}^{(0)}(\theta)} .
$$

For the quenched microscopic density we thus need the effective partition function for $N_{f}=2$, which fortunately is known in analytical form (see eq. (23)). For simplicity taking the case of $\theta=0$, we indeed find from eq. (23) that

$$
\rho_{S}(\lambda ; \theta=0)=\frac{1}{2 \mathcal{Z}^{(0)}}|\zeta| .
$$

The density consistently comes out linear, and again with an undetermined constant in front. To determine this constant we would normally impose the matching condition of $\rho_{S}(\zeta \rightarrow \infty)=1 / \pi$, an impossible requirement in this case. Again, the failure of these attempts at taking the quenched limit in this framework is perhaps not surprising. The presumed distribution (51) concerns by construction only the pure gauge sector, for which no information can be gained via the (anomalous) chiral Ward identities on which the present framework is based. Moreover, in the pure gauge theory we can sum over gauge field configurations with also fractional winding numbers [25]. In the large- $N_{c}$ limit the topological susceptibility in the quenched theory is, via the Witten-Veneziano relation [29], related to the flavor singlet $\eta^{\prime}$ mass. In the quenched limit, where the anomaly plays no role, the $\eta^{\prime}$ should be treated as a (pesudo)-Goldstone boson too. Perhaps an analogue of the large- $N_{c}$ effective Lagrangian with built-in information about the topological susceptibility of the pure gauge theory (as required by the Witten-Veneziano relation, at least at large $N_{c}$ ) can be used to treat this case in a consistent manner.

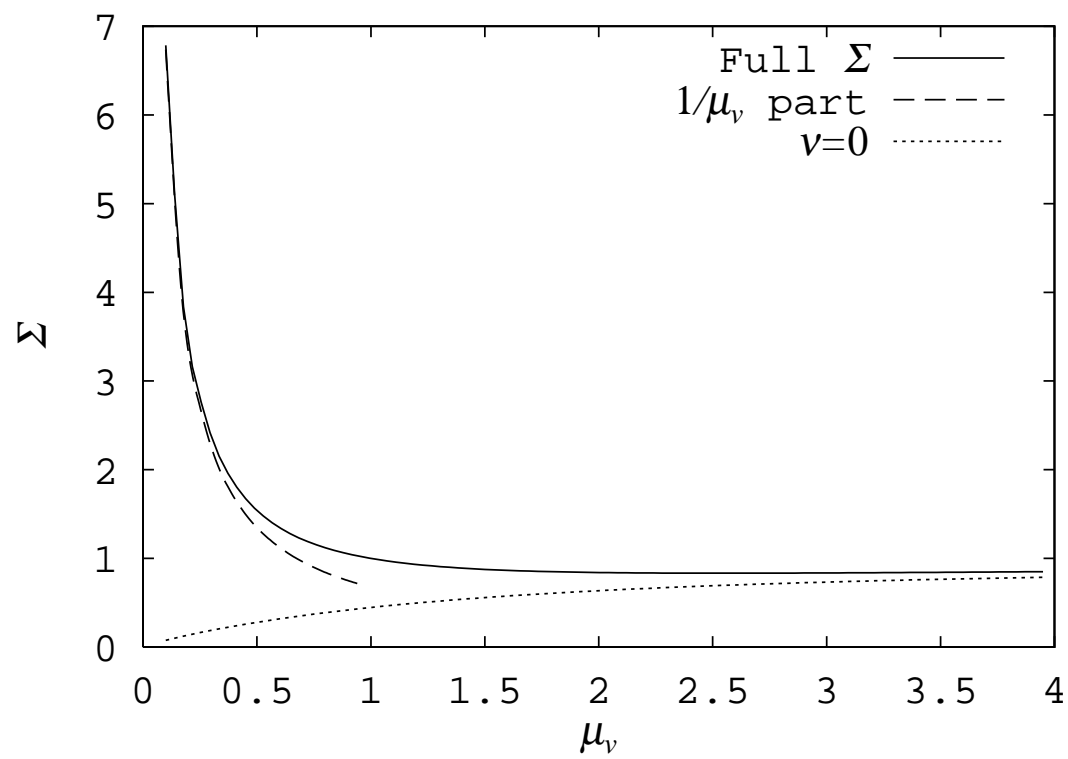

Figure 8: The partially quenched chiral condensate $\Sigma\left(\mu_{v}, \mu\right)$ in the $N_{f}=1$ theory with $\theta=0$. The "quenched" mass is indicated by $\mu_{v}$, while $\mu$ is the real physical mass, here taken to be unity for convenience. The sum over topological charges converges everywhere, except at the point $\mu_{v}=0$. In this case the divergence at $\mu_{v} \rightarrow 0$ can easily be worked out analytically (see eq. (59)), and for comparison we display also just the resulting $1 / \mu_{v}$ term with the coefficient as given in eq. (59).

There are no such difficulties with partial quenching. For example, the dependence of the partially quenched chiral condensate on the (quenched) "valence quark" mass $\mu_{v}$ is computed in the full theory 
by averaging with respect to the usual fixed- $\nu$ partition function $\mathcal{Z}_{\nu}$. This sum converges for $\mu_{v} \neq 0$. To be concrete, consider the analytical expression for the $N_{f}=1$ case which was derived in the second of ref. [15]:

$$
\begin{aligned}
\Sigma_{\nu}\left(\mu_{v}, \mu\right)= & \mu_{v}\left[I_{\nu+1}\left(\mu_{v}\right) K_{\nu+1}\left(\mu_{v}\right)+I_{\nu+2}\left(\mu_{v}\right) K_{\nu}\left(\mu_{v}\right)\right] \\
& +2 \mu \frac{K_{\nu}\left(\mu_{v}\right)}{I_{\nu}(\mu)} \frac{\mu_{v} I_{\nu}\left(\mu_{v}\right) I_{\nu+1}(\mu)-\mu I_{\nu}(\mu) I_{\nu+1}\left(\mu_{v}\right)}{\mu_{v}^{2}-\mu^{2}}+\frac{\nu}{\mu_{v}}
\end{aligned}
$$

In fig. 8 we show this as a function of $\mu_{v}$ for $\nu=0$ (which of course does not exhibit the $1 / \mu_{v^{-}}$ singularity), and fixed value of the physical "sea quark" mass $\mu$. We also show the full answer, for the same mass $\mu$, obtained by summing over winding numbers according to

$$
\Sigma\left(\theta ; \mu_{v}, \mu\right)=\mathcal{Z}(\theta ; \mu)^{-1} \sum_{\nu=-\infty}^{\infty} e^{i \nu \theta} \mathcal{Z}_{\nu}(\mu) \Sigma_{\nu}\left(\mu_{v}, \mu\right)
$$

where in this case $\mathcal{Z}(\theta ; \mu)=\exp [\mu \cos \theta]$, and $\mathcal{Z}_{\nu}(\mu)=I_{\nu}(\mu)$.

The partially quenched cases are particularly interesting because they provide a limit in which the $\sim 1 / \mu_{v}$ divergences of the quenched approximations are under full control from the analytical point of view. Indeed, the coefficient of the $1 / \mu_{v}$-term in the partially quenched $N_{f}=1$ theory can be computed analytically in this finite-volume limit; it equals

$$
\langle\langle|\nu|\rangle\rangle=\mu e^{-\mu}\left(I_{0}(\mu)+I_{1}(\mu)\right) .
$$

This curve has also been included in fig. 8 , and one clearly sees how accurately it describes the $1 / \mu_{v}$ singularity.

\subsection{EXTENSIONS}

The present results easily extend to the other two major classes of chiral symmetry breaking [12]. The analytically most simple case is that corresponding to gauge group $\mathrm{SU}\left(N_{c} \geq 3\right)$ and $N_{f}$ fermions in the adjoint representation (which in Random Matrix Theory language corresponds to the chSE). For example, from the relations derived in the second of reference 13 we know that we in this case can express the microscopic spectral density of the Dirac operator in a sector of topological charge $\nu$ by

$$
\rho_{S}^{(\nu)}\left(\zeta ;\left\{\mu_{i}\right\}\right)=C_{4} \zeta^{3} \frac{\mathcal{Z}_{\nu}^{\left(N_{f}+4\right)}\left(\left\{\mu_{i}\right\},\{i \zeta\}\right)}{\mathcal{Z}_{\nu}^{\left(N_{f}\right)}\left(\left\{\mu_{i}\right\}\right)} .
$$

Also this can be summed over topological charge $\nu$ to get the full microscopic spectral density for that case. Using a similar relation for all higher $k$-point functions [13]

$$
\begin{aligned}
\rho_{S}^{(\nu)}\left(\zeta_{1}, \ldots, \zeta_{k} ;\left\{\mu_{i}\right\}\right)= & C_{4}^{(k)} \prod_{i}^{k}\left(\zeta_{i}^{3} \prod_{f}^{N_{f}}\left(\zeta_{i}^{2}+\mu_{f}^{2}\right)\right) \prod_{j<l}^{k}\left|\zeta_{j}^{2}-\zeta_{l}^{2}\right|^{4} \\
& \times \frac{\mathcal{Z}_{\nu}^{\left(N_{f}+4 k\right)}\left(\left\{\mu_{i}\right\} ;\left\{i \zeta_{1}\right\}, \ldots,\left\{i \zeta_{k}\right\}\right)}{\mathcal{Z}_{\nu}^{\left(N_{f}\right)}\left(\left\{\mu_{i}\right\}\right)}
\end{aligned}
$$

also these $k$-point functions can be averaged over topological charges to yield a ratio of two full partition functions, without restrictions to topology. In these relation each (imaginary) additional fermion mass 
on the right hand side is four-fold degenerate. Unfortunately, the relevant fixed- $\nu$ partition functions are not in general known in closed analytic form, and the proportionality constants in both of the above relations have therefore not yet been determined. Until this has been done, and in particular their $\nu$-dependences have been found, we cannot explicitly identify the combination of finite-volume partition functions that will emerge from the sums.

\section{Conclusions}

We have here considered the effect of summing over all topological charges $\nu$, while remaining in the finite-volume scaling regime defined by eq. (11). For the mass-dependent chiral condensate the effect of summing over topological sectors is quite drastic. For example, the condensate actually diverges like $\sim 1 / \mu$ in the $\mu \rightarrow 0$ limit if one restricts oneself to any sector of non-vanishing topological charge $\nu$, even though the full condensate vanishes as $\mu \rightarrow 0$ (except for the case $N_{f}=1$ ). The microscopic Dirac operator spectrum itself is also substantially modified by the summation over topological charges. A simple compact relation gives the full microscopic spectral density in terms of a ratio of partition functions, one of which is evaluated at a shifted vacuum angle $\theta \rightarrow \theta+\pi$, and with two additional fermion species. We have pointed out a difficulty with obtaining the quenched $\left(N_{f} \rightarrow 0\right)$ limit in this simple way. The difficulty disappears if one focuses instead on a partially quenched limit, in which the appropriate weight factors in the sum over topological numbers again are known exactly in this finite-volume regime. We have also noted a drastic simplification in the massless case, where the full microscopic spectral density actually coincides with the one of the sector with $\nu=0$.

An interesting open question is whether one can establish a compact Random Matrix Theory formulation also for the full effective partition function $\mathcal{Z}\left(\theta ;\left\{\mu_{i}\right\}\right)$. Although we believe the answer to be negative, we are not aware of any strong argument to that effect.

In future lattice studies it seems obvious to try to study not just the microscopic Dirac operator spectrum in sectors of fixed $\nu$, but also the associated distribution of configurations labelled by the topological index. Thanks to very recent developments this is now possible [21, 22]. This way one can test simultaneously the probability distribution of configurations with fixed topological index (which is known analytically in the regime (1) and given, for $\theta=0$, simply by $\left.\mathcal{Z}_{\nu}\left(\left\{\mu_{i}\right\}\right) / \mathcal{Z}\left(\theta=0 ;\left\{\mu_{i}\right\}\right)\right)$, and, simultaneously, the full microscopic Dirac operator spectrum. Such numerical simulations with dynamical fermions now seem feasible.

Acknowledgements: Discussions with Urs Heller and Jac Verbaarschot are gratefully acknowledged. This work has been partially supported by EU TMR grant no. ERBFMRXCT97-0122.

\section{References}

[1] J. Gasser and H. Leutwyler, Phys. Lett. B188 (1987) 477.

[2] D. Weingarten, Phys. Rev. Lett. 51 (1983) 2351.

[3] H. Leutwyler and A. Smilga, Phys. Rev. D46 (1992) 5607. 
[4] A. Smilga and J.J.M. Verbaarschot, Phys. Rev D51 (1995) 829.

M.A. Halasz and J.J.M. Verbaarschot, Phys. Rev D52 (1995) 2563.

[5] E.V. Shuryak and J.J.M. Verbaarschot, Nucl. Phys. A560 (1993) 306.

[6] J.J.M. Verbaarschot and I. Zahed, Phys. Rev. Lett. 70 (1993) 3852; Phys. Rev. Lett. 73 (1994) 2288.

J.J.M. Verbaarschot, Phys. Lett. B329 (1994) 351; Nucl. Phys. B426 (1994) 559.

[7] G. Akemann, P.H. Damgaard, U. Magnea and S. Nishigaki, Nucl. Phys. B487 (1997) 721; Nucl. Phys. B519 (1998) 682.

[8] P.H. Damgaard and S.M. Nishigaki, Nucl. Phys. B518 (1998) 495.

[9] P.H. Damgaard and S.M. Nishigaki, Phys. Rev. D57 (1998) 5299.

J. Christiansen, hep-th/9809194.

[10] M.K. Şener and J.J.M. Verbaarschot, Phys. Rev. Lett. 81 (1998) 248.

[11] K. Splittorff, hep-th/9810248.

[12] J.J.M. Verbaarschot, Phys. Rev. Lett. 72 (1994) 2531.

[13] P.H. Damgaard, Phys. Lett. B424 (1998)322.

G. Akemann and P.H. Damgaard, Nucl. Phys. B519 (1998) 682; Phys. Lett. B432 (1998) 390.

S.M. Nishigaki, P.H. Damgaard and T. Wettig, Phys. Rev. D58 (1998) 087704.

[14] P.H. Damgaard, hep-th/9807026.

[15] J.C. Osborn, D. Toublan and J.J.M. Verbaarschot, hep-th/9806110.

P.H. Damgaard, J.C. Osborn, D. Toublan and J.J.M. Verbaarschot, hep-th/9811212.

[16] J. Smit and J.C. Vink, Nucl. Phys. B286 (1987) 485.

[17] J.J.M. Verbaarschot, Phys. Lett. B368 (1996) 137.

[18] M.E. Berbenni-Bitsch, S. Meyer, A. Schäfer, J.J.M. Verbaarschot and T. Wettig, Phys. Rev. Lett. 80 (1998) 1146.

M.E. Berbenni-Bitsch, S. Meyer, T. Wettig, Phys. Rev. D58 (1998) 071502.

[19] P.H. Damgaard, U.M. Heller and A. Krasnitz, Phys. Lett. B445 (1999) 366.

M. Göckeler, H. Hehl, P.E.L. Rakow, A. Schäfer and T. Wettig, hep-lat/9811018.

R.G. Edwards, U.M. Heller and R. Narayanan, hep-lat/9902021.

[20] P. Chen et al., hep-lat/9807029.

R.G. Edwards, U.M. Heller and R. Narayanan, hep-lat/9811030 (to appear in Phys. Rev. D).

[21] F. Farchioni, I. Hip, C.B. Lang and M. Wohlgenannt, hep-lat/9812018.

[22] R.G. Edwards, U.M Heller, J. Kiskis and R. Narayanan, hep-th/9902117.

[23] R.A. Janik, M.A. Nowak, G. Papp and I. Zahed, hep-ph/9901390.

[24] R. Brower, P. Rossi and C.-I. Tan, Nucl. Phys. B190[FS3] (1981) 699.

A.D. Jackson, M.K. Şener and J.J.M. Verbaarschot, Phys. Lett. B387 (1996) 355. 
[25] G. 't Hooft, Commun. Math. Phys. 81 (1981) 267.

[26] P.H. Damgaard, Phys. Lett. B425 (1998) 151.

[27] M. Lüscher, Nucl. Phys. B205[FS5] (1982) 483.

[28] R.J. Crewther, Phys. Lett. B70 (1977) 349.

[29] E. Witten, Nucl. Phys. B156 (1979) 269.

G. Veneziano, Nucl. Phys. B159 (1979) 213. 